\title{
How would the introduction of an EU-wide formula apportionment affect the distribution and size of the corporate tax base? An analysis based on German multinationals
}

\author{
Clemens Fuest • Thomas Hemmegarn • Fred Ramb
}

Published online: 11 July 2007

(C) Springer Science+Business Media, LLC 2007

Erratum to: Int Tax Public Finance

DOI 10.1007/s10797-006-9008-6

Tables 1-4 are incorrect in both online and print versions of this article. The decimal separators should have been commas. New tables follow on next pages.

The online version of the original article can be found at http://dx.doi.org/10.1007/s10797-006-9008-6.

C. Fuest · T. Hemmegarn ( $\varangle)$

Department of Economics, University of Cologne, Albertus-Magnus-Platz, 50923 Cologne, Germany e-mail: thomas.hemmelgarn@uni-koeln.de

F. Ramb

Deutsche Bundesbank, Economic Research Centre, Wilhelm-Epstein-Straße 65439, e14, 60431 Frankfurt am Main, Germany 
Table 1 The mean of profit, property, sales (in thousand Euro) and number of employees for the years 1996-2001

\begin{tabular}{lcclrr}
\hline Country & & & & & $\begin{array}{c}\text { Number } \\
\text { of firms }\end{array}$ \\
& mprofit & mproperty & msales & memployees & \\
Austria & & & & & \\
Belgium & $818(22,114)$ & $8,880(45,412)$ & $46,688(156,264)$ & $173(497)$ & 702 \\
Denmark & $3,372(28,086)$ & $11,417(66,109)$ & $71,602(355,387)$ & $156(778)$ & 427 \\
Finland & $245(6,649)$ & $4,344(16,095)$ & $29,076(69,128)$ & $100(237)$ & 208 \\
France & $1,345(5,964)$ & $8,107(38,744)$ & $22,581(56,847)$ & $68(177)$ & 195 \\
Germany & $1,050(49,848)$ & $10,427(52,142)$ & $90,259(653,058)$ & $230(868)$ & 993 \\
Great Britain ${ }^{*}$ & $8,560(156,078)$ & $66,202(596,773)$ & $378,836(2,164,687)$ & $1,686(9,483)$ & 1,844 \\
Greece & $-10,210(223,247)$ & $17,295(135,137)$ & $80,785(510,215)$ & $245(1,575)$ & 790 \\
Ireland & $-9(2,503)$ & $4,423(7,831)$ & $27,604(47,498)$ & $108(174)$ & 99 \\
Italy & $2,503(14,733)$ & $5,560(18,540)$ & $17,103(39,054)$ & $90(194)$ & 110 \\
Luxemburg ${ }^{*}$ & $1,999(14,349)$ & $9,551(43,574)$ & $56,082(243,091)$ & $137(392)$ & 609 \\
The Netherlands & $-4,973(158,101)$ & $11,115(40,902)$ & $26,068(58,859)$ & $83(154)$ & 95 \\
Portugal & $6,954(162,486)$ & $9,834(53,486)$ & $35,800(102,136)$ & $109(238)$ & 611 \\
Spain & $1,267(10,817)$ & $17,314(84,595)$ & $49,121(189,388)$ & $225(526)$ & 186 \\
Sweden & $2,415(17,554)$ & $11,174(56,575)$ & $55,832(255,940)$ & $207(745)$ & 545 \\
\hline & $3,717(41,394)$ & $20,056(158,259)$ & $68,260(379,808)$ & $225(1,498)$ & 256 \\
\hline
\end{tabular}

Standard deviations in parenthesis.

* The negative values for these countries are due to large losses of single firms during the sample period.

Table 2 The sum of the SA tax base for the EU15 countries in m. Euro for the years 1996-2001

\begin{tabular}{lc}
\hline Country & SA tax base \\
\hline Austria & 6,144 \\
Belgium & 7,659 \\
Denmark & 972 \\
Finland & 676 \\
France & 16,056 \\
Germany & 114,700 \\
Great Britain & 8,549 \\
Greece & 379 \\
Ireland & 1,314 \\
Italy & 6,634 \\
Luxemburg & 1,363 \\
The Netherlands & 21,460 \\
Portugal & 1,923 \\
Spain & 6,897 \\
Sweden & 4,691 \\
\hline
\end{tabular}


Table 3 Comparison between the sum of the SA and an FA tax base without international loss-offset for the EU15 countries in m. Euro for the years 1996-2001

\begin{tabular}{lrrrr}
\hline Country & SA tax base & $\begin{array}{l}\text { Formula apportionment } \\
\text { without loss-offset }\end{array}$ & Difference & $\begin{array}{l}\text { Change in } \\
\text { percent (\%) }\end{array}$ \\
\hline Austria & 6,144 & 16,793 & 10,649 & 173 \\
Belgium & 7,659 & 5,625 & $-2,034$ & -27 \\
Denmark & 972 & 1,047 & 75,309 & 8 \\
Finland & 676 & 635 & -41 & -6 \\
France & 16,056 & 16,129 & 73 & 0 \\
Germany & 114,700 & 121,400 & 6,714 & 6 \\
Great Britain & 8,549 & 13,168 & 4,619 & 54 \\
Greece & 379 & 527 & 148 & 39 \\
Ireland & 1,314 & 793 & -521 & -40 \\
Italy & 6,634 & 6,748 & 114 & 2 \\
Luxembourg & 1,363 & 1,114 & -249 & -18 \\
The Netherlands & 21,460 & 7,543 & $-13,916$ & -65 \\
Portugal & 1,923 & 2,666 & 744 & 39 \\
Spain & 6,897 & 7,273 & 377 & 5 \\
Sweden & 4,691 & 3,053 & $-1,639$ & -35 \\
\hline
\end{tabular}

Table 4 Comparison of the sum of the SA tax base and the FA tax base for the years 1996-2001 in m. Euro

\begin{tabular}{|c|c|c|c|c|}
\hline Country & SA tax base & FA tax base & Difference & $\begin{array}{l}\text { Change in } \\
\text { percent }(\%)\end{array}$ \\
\hline Austria & 6,144 & 13,005 & 6,861 & 112 \\
\hline Belgium & 7,659 & 4,550 & $-3,109$ & -41 \\
\hline Denmark & 972 & 737 & -235 & -24 \\
\hline Finland & 676 & 509 & -167 & -25 \\
\hline France & 16,056 & 11,011 & $-5,045$ & -31 \\
\hline Germany & 114,700 & 95,351 & $-19,313$ & -17 \\
\hline Great Britain & 8,549 & 7,869 & -680 & -8 \\
\hline Greece & 379 & 435 & 56 & 15 \\
\hline Ireland & 1,314 & 650 & -664 & -51 \\
\hline Italy & 6,634 & 4,882 & $-1,752$ & -26 \\
\hline Luxemburg & 1,363 & 829 & -534 & -39 \\
\hline The Netherlands & 21,460 & 5,481 & $-15,979$ & -74 \\
\hline Portugal & 1,923 & 2,165 & 242 & 13 \\
\hline Spain & 6,897 & 5,777 & $-1,120$ & -16 \\
\hline Sweden & 4,691 & 2,004 & $-2,685$ & -57 \\
\hline EU15 & 199,400 & 155,300 & $-44,100$ & -22 \\
\hline
\end{tabular}

